\title{
Assessment and follow up of patients prescribed long term oxygen treatment
}

\author{
L J Restrick, E A Paul, G M Braid, P Cullinan, J Moore-Gillon, J A Wedzicha,
}

\begin{abstract}
Background-Prescription and use of long term oxygen treatment were audited in a large group of patients after more than five years of use of the guidelines for its prescription.

Methods-Patients with a concentrator were interviewed at home with a structured questionnaire in three family health service authorities in East London. Stable oxygen saturation $\left(\mathrm{SaO}_{2}\right)$ breathing air and oxygen, forced expiratory volume in one second $\left(F E V_{1}\right)$ and current and previous dated concentrator meter readings were recorded. A further questionnaire was sent to each patient's general practitioner. Hospital case notes of patients who did not meet the criteria for long term oxygen treatment at reassessment were reviewed.
\end{abstract}

Results-A total of 176 patients were studied; $84 \%$ had chronic obstructive lung disease and $19 \%$ admitted to continued smoking; 140 patients had seen a respiratory physician but results of respiratory assessment were available to their general practioner in fewer than 54 cases. $F_{1} V_{1}$ was $<1.51$ in 158 patients but in $67 \mathrm{SaO}_{2}$ was less than $91 \%$ breathing air, mainly in patients with chronic obstructive lung disease who had been inadequately assessed. Daily oxygen was prescribed for a median of 15 (range 4-24) hours and measured daily use was 15 (0-24) hours; $74 \%$ of patients used more than 12 hours. Only 35 patients had problems with oxygen treatment, but 29 had an undercorrected $\mathrm{SaO}_{2}$ of less than $92 \%$ when using their concentrator.

Conclusions-Guidelines for prescription of long term oxygen treatment are largely followed and most patients complied with treatment. Increased communication about respiratory state is required between hospital doctors and general practitioners. Patients need regular reassessments to ensure that hypoxaemia is corrected and that oxygen is appropriately prescribed.

(Thorax 1993;48:708-713)

In December 1985, after the publication of a Medical Research Council study and the
Nocturnal Oxygen Therapy Trial $^{12}$ showing that long term oxygen treatment improved survival in patients with hypoxic chronic obstructive lung disease, the Department of Health produced guidelines for the prescription of oxygen concentrators by general practitioners. The indications for prescribing long term oxygen are described in the Appendix. Current guidelines recommend that all patients should be assessed when their condition is stable by a respiratory physician with verification of results after three weeks, before long term oxygen treatment is recommended. ${ }^{3}$ The guidelines also include recommendations for follow up, including a statement on the requirement for "regular monitoring in the home by appropriately trained nurses or lung function technicians to ensure that treatment is achieving optimum results." There is, however, currently no provision for formal organisation of this domiciliary follow up.

Earlier surveys in England of small numbers of patients (ranging from 61 to 82 ) have each described a different aspect of concentrator prescription and use. ${ }^{4-6}$ Initial prescription of oxygen did not always adhere to the guidelines and was often inappropriate, particularly when the prescription was initiated by a general practitioner without previous specialist respiratory assessment. ${ }^{45}$ In both the United Kingdom ${ }^{6}$ and Europe ${ }^{7}$ long term oxygen treatment has had problems, either because of inappropriate instructions or because of poor compliance. As the guidelines for prescribing long term oxygen have now been in use for more than five years it is useful to audit the current situation.

We assessed both prescription and use of long term oxygen in a large group of patients. The most important issues addressed were adherence to the guidelines for prescription, communication between respiratory physicians and general practitioners, and the provision of follow up, which have not previously been studied; patient compliance and problems with the use of long term oxygen; and the characteristics of patients who, at domiciliary reassessment, did not meet the criteria for oxygen treatment.

\section{Methods}

All patients recorded as having a concentrator on 1 January 1991 in the three family health 
service authorities of City and East London, Waltham Forest and Redbridge, and Barking and Havering were studied. All patients were visited at home between 1 February 1991 and 1 May 1991. Their respiratory function was measured and they were interviewed using a structured questionnaire. Stable oxygen saturation $\left(\mathrm{SaO}_{2}\right)$ after 20 minutes breathing room air and after 20 minutes breathing oxygen delivered by the concentrator, at the setting used by the patient, were recorded with pulse oximeters (Pulsox-7, Pulsox-8, Minolta; De Vilbiss Health Care UK, Heston, Middlesex). Forced expiratory volume in one second $\left(\mathrm{FEV}_{1}\right)$ was measured with the Morgan dry spirometer or Micro spirometer (Micro Medical, Chatham, Kent) and the best of three measurements recorded.

The interviewer used a questionnaire to gain information on the initial prescription of oxygen; the instructions given about using the concentrator; outpatient follow up; current symptoms, particularly breathlessness, level of activity, and mobility including whether house bound and whether limited by breathlessness or other symptoms; social situation including whether living alone with or without ground floor access; smoking state; use of the concentrator and availability of portable oxygen; and problems with the treatment.

To obtain a measure of the daily number of hours of oxygen treatment, the current and previous dated meter readings were used to calculate total meter use. Actual daily hours of use were calculated, with the number of nights the patients had been away from home since the last meter reading and the hours in each day when the machine was switched on but not in use being taken into account.

A further questionnaire was sent to the general practitioner of each patient in the study seeking details of the patient's diagnosis, prescription, and respiratory assessment (if any) for oxygen treatment. Hospital case notes of patients whose $\mathrm{SaO}_{2}$ was above $91 \%$ while breathing air ${ }^{8}$ at the home visit were traced. Information on their initial assessment and prescription of oxygen and details of any follow up were retrieved.

Though age is not mentioned in the guidelines for the prescription of oxygen, ${ }^{3}$ doubts have been expressed about the benefits of prescribing oxygen to elderly people. Symptoms, compliance, and problems with the treatment were therefore compared, as indicators of the potential benefit of oxygen, between patients under 75 and those of 75 and older. Results were also analysed for differences in prescription and compliance between the three family health service authorities.

\section{STATISTICAL ANALYSIS}

Data analysis and descriptive statistics were performed with the statistical package for the social sciences (SPSS). Hours of oxygen concentrator use, oxygen flow rate and duration of installation are expressed as medians and ranges. Arterial oxygen tension $\left(\mathrm{PaO}_{2}\right)$, arterial carbon dioxide tension $\left(\mathrm{PaCO}_{2}\right), \mathrm{FEV}_{1}$, forced vital capacity (FVC), and $\mathrm{SaO}_{2}$ are expressed as means and standard deviations. A Student's paired $t$ test was used to compare $\mathrm{SaO}_{2}$ on and off the concentrator; $\chi^{2}$ tests, two sample $t$ tests, and Mann-Whitney tests were used as appropriate to compare differences in compliance between those under 75 and those 75 and older, and differences in prescription and use between the family health service authorities.

\section{Results}

There were 191 patients with an oxygen concentrator in the three family health service authorities studied; 141 in City and East London, 27 in Waltham Forest and Redbridge, and 23 in Barking and Havering. The three family health service authorities have a total population of 1150000 , giving an overall age adjusted rate of prescription of 16 per 100000 population. Overall, 180 patients out of 191 were available for assessment. Of the remaining 11 patients, eight died before home assessment was performed, one patient received a lung transplant, one patient had moved out of the area, and in one case the concentrator had been removed for underuse. Home assessments were performed in 176 patients (98\%). The four patients who did not have home assessments included two who worked regularly, one who had a prolonged spell in hospital during the period of study, and one who refused. A total of 173 (96\%) replies were received from the postal questionnaire to general practitioners.

\section{PATIENT CHARACTERISTICS}

Table 1 lists the characteristics of the patients studied. A previous episode of cor pulmonale was described for $38 \%(56 / 148)$ of the patients with chronic obstructive lung disease. Most of the patients studied had symptoms of breathlessness; $78 \%$ (137) said that they were breathless walking around at home and $24 \%(42 / 172)$ were observed to be

Table 1 Characteristics of patients $(n=176)$. Values are numbers (percentages) of patients unless stated otherwise

\begin{tabular}{lc}
\hline Characteristic & \\
\hline Sex ratio (M:F) & $92: 84$ \\
Median age (range) (years) & $67(28-87)$ \\
Median duration of concentrator & $19(1-64)$ \\
$\quad$ installation (range) (months) & \\
Concentrator use (years): & \\
$>1$ & $113(64)$ \\
$>2$ & $66(38)$ \\
$>3$ & $34(19)$ \\
Smokers & $34(19)$ \\
Diagnosis: & $148^{\star}(84)$ \\
Chronic obstructive lung disease & $9(5)$ \\
Pulmonary fibrosis & $5(3)$ \\
Bronchiectasis & $6(3)$ \\
Nocturnal hypoventilation/apnoea & $2(1)$ \\
Heart disease & $1(1)$ \\
Lymphoma & $1(1)$ \\
Lymphangiomyomatosis & $4(2)$ \\
Not available & $162(92)$ \\
Attended outpatient clinics &
\end{tabular}

^Nine also had restrictive lung disease and two carcinoma of the lung. 
breathless on talking. However, 55\% (97) of patients said that they were able to go out (other than for medical visits to their general practitioner or to hospital), including $47 \%$ $(65 / 137)$ who said that they were breathless walking around at home. Only 9\% (16) of patients had reasons other than breathlessness which limited mobility. In all, $22 \%$ (39) of patients lived alone and $68 \%(115 / 168)$ had ground floor access to their homes. Of the patients who did not otherwise go out, $87 \%(69 / 79)$ still attended outpatient clinics.

\section{INITIAL ASSESSMENT FOR PRESCRIPTION}

In all, 140 patients had been seen by a respiratory physician who recommended the prescription of long term oxygen; eight prescriptions were recommended by other hospital physicians and 19 were initiated by the general practitioner. For 13 patients the initiator of the prescription was not known. A total of 132 patients were known by their general practitioner to have had a formal respiratory assessment, but the results of $\mathrm{PaO}_{2}$ measurements were available to the general practitioner in only 54 cases (mean (SD) $\left.\mathrm{PaO}_{2} 6.5(1.4) \mathrm{kPa}\right)$; the results of $\mathrm{PaCO}_{2}$ were available in only 42 cases $(6.9(1.4) \mathrm{kPa})$. The general practitioner knew of the $\mathrm{FEV}_{1}$ and FVC measured at assessment in even fewer cases, 33 and 32 respectively, with the mean $\mathrm{FEV}_{1}$ being $0.8(0.4) 1$ and FVC 1.7 $(0 \cdot 7) 1$.

\section{ASSESSMENT AT STUDY INTERVIEW}

\section{Spirometry}

$\mathrm{FEV}_{1}$ was below 1.51 in 158 patients, with a mean of $0.52(0.32) 1$ and mean FVC was $1.30(0.63) 1$.

Figure (A) Daily hours of prescribed use of oxygen $(n=156)$. (B) Daily hours of actual use of oxygen $(n=175)$.
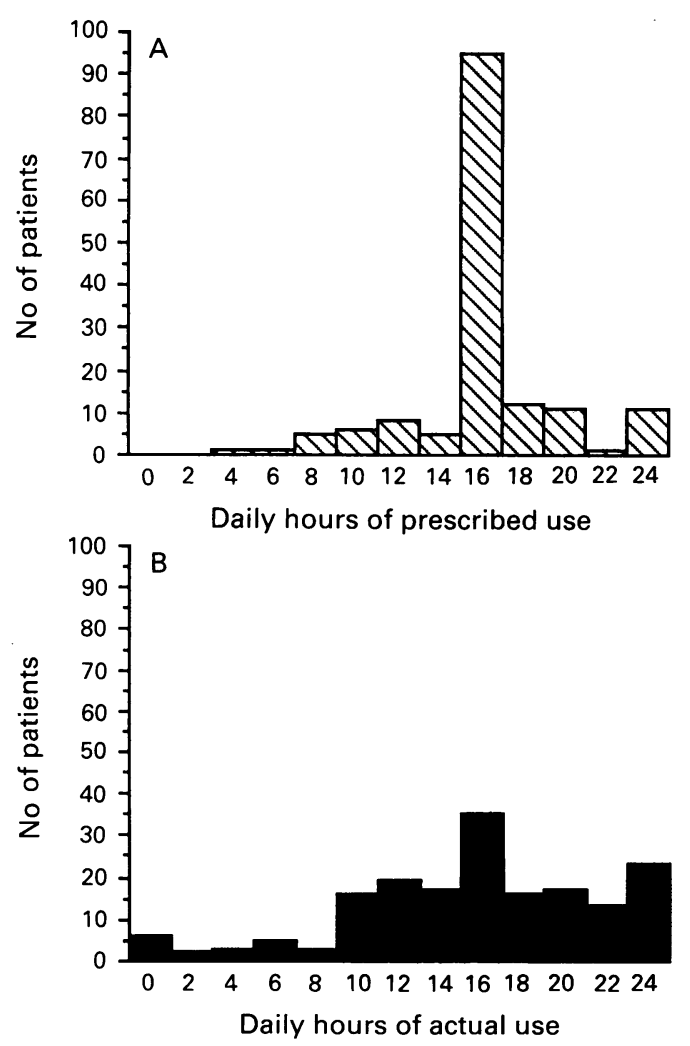

Oximetry

In the 173 patients in whom it was possible to measure $\mathrm{SaO}_{2}$ after 20 minutes of breathing air (three felt too unwell without oxygen) the mean (SD) $\mathrm{SaO}_{2}$ was $88.9 \%(5 \cdot 8 \%)$. One hundred and six (61\%) patients had an $\mathrm{SaO}_{2}$ of $91 \%$ or below when breathing air at reassessment and were considered to fulfil the guidelines for long term oxygen at follow up. The mean $\mathrm{SaO}_{2}$ after 20 minutes of breathing oxygen delivered by the concentrator measured in 170 patients was $94.4 \%$ (3.4\%). $\mathrm{SaO}_{2}$ was significantly increased by using the concentrator compared with breathing air ( $p<0.005 ; 95 \%$ confidence interval of difference $5 \cdot 1 \%$ to $6 \cdot 3 \%$ ). However, 29 patients had an undercorrected $\mathrm{SaO}_{2}$ of less than $92 \%$ when using their concentrator to deliver oxygen even though 28 of these patients were under supervision as outpatients.

\section{PRESCRIPTION AND USE}

The figure (A) shows the histogram for hours of daily use of oxygen prescribed by the general practitioner. The median number of hours prescribed was 15 (range 4-24 hours), and $83 \%(130 / 156)$ of patients were prescribed treatment for 15 hours or more in accordance with the guidelines. Twenty seven percent (47) of patients acknowledged that for a median period of two hours (range $0 \cdot 5-23$ hours) they did not wear the cannulae or a mask when the concentrator was switched on (non-use). When this non-use was accounted for the median stated daily use by patients was $16(0-24)$ hours. The figure (B) shows the histogram for hours of daily measured use calculated from meter readings, with nights away from home and non-use taken into account. The median measured use was 15(0-24) hours, with 74\% (130/175) of patients having a daily measured use of more than 12 hours. Seventeen $(10 \%)$ of patients said that they had difficulty achieving the number of hours prescribed each day and for $33 \%(58 / 175)$ of patients the measured use was at least two hours less than the number of hours the patients said that the concentrator was switched on. There were no significant differences in adherence to the prescription guidelines or compliance with treatment between the three family health service authorities.

The median flow rate recorded for 125 patients was $2 \mathrm{l} / \mathrm{min}$ (1-8 $1 / \mathrm{min})$. Overall, 158 patients used nasal cannulae, 18 a mask, and five both cannulae and a mask. Only 27 patients had a humidifier fitted. Forty patients had a portable oxygen cylinder, of whom 22 said they were able to go out, and two patients had transtracheal oxygen catheters.

Overall, 98\% (164/167) of patients said that they found oxygen treatment helpful, thirty five patients described problems related either to the concentrator (noise was identified as a problem by 27 patients and sleep disturbance by one patient) or to the method of delivery (five patients complained of restricted movement, four of nasal discomfort, two of ear discomfort, and two of social 
embarrassment). The patients with problems were no less compliant with taking treatment and had adequately corrected oxygen saturations with oxygen, the mean $\mathrm{SaO}_{2}$ using the concentrator being $94 \cdot 7 \%(2 \cdot 9 \%)$.

Thirty four patients were aged 75 and over; $76 \%(26)$ of these were breathless walking round at home and $47 \%$ (16) were able to go out. The median hours of daily use (14 (1-24)) was significantly less in those of 75 and older than in those under $75^{\prime}(16(0-24))$ $(p=0.005)$, but the proportion with problems with the treatment was not significantly different between the two age groups (21\% $(7 / 34)$ in those of 75 and over and $20 \%$ $(28 / 142)$ in the younger patients).

REVIEW OF INITIAL PRESCRIPTION IN PATIENTS WITH $\mathrm{SaO}_{2}$ ABOVE $91 \%$ WHEN BREATHING AIR AT STUDY INTERVIEW

At follow up 67 patients had an $\mathrm{SaO}_{2}$ above $91 \%$ when breathing air. These patients were studied in further detail to evaluate whether they fulfilled the criteria for long term oxygen treatment at the time of initial prescription. Information was available on $58(87 \%)$ of these patients, from case notes for 55 patients, and from the general practitioner questionnaire for the three patients who were under the care of their family doctor alone. Table 2 shows the characteristics of these patients.

All 13 of the patients with nocturnal hypoventilation had been fully assessed for long term oxygen, including by overnight sleep studies. Only one of each of the two patients with bronchiectasis and heart failure had been formally assessed and met the criteria for its prescription. Of the 41 patients with chronic obstructive lung disease, 30 were prescribed oxygen on the instructions of a respiratory physician. However, in seven of these patients assessment was performed during an exacerbation when the patient's condition was not stable, and two patients were not assessed formally. The mean $\mathrm{PaO}_{2}$ at the time of prescription in the 23 of the 30 in whom it was available was $7.6(1.4) \mathrm{kPa}$ and 11 patients had a $\mathrm{PaO}_{2}$ above $7 \cdot 3 \mathrm{kPa}$. Twenty nine of the 30 patients in whom oxygen treatment was initiated by a respiratory physician

Table 2 Characteristics of patients with $\mathrm{SaO}_{2}>91 \%$ breathing air at study interview $(n=58)$. Values are numbers (percentages) of patients unless stated otherwise

\begin{tabular}{ll}
\hline Sex ratio (M:F) & $35: 23$ \\
Median age (range) (years) & $68(28-87)$ \\
Prescription by respiratory physician & $45(78)$ \\
Respiratory assessment performed & 37 \\
Prescription by general practitioners & $13(22)$ \\
Respiratory assessment performed & 0 \\
Diagnosis: & $41(71)$ \\
Chronic obstructive lung disease & $13(23)$ \\
Nocturnal hypoventilation & 5 \\
Chronic obstructive lung disease & 4 \\
Chest wall/neuromuscular disease & 2 \\
Obstructive sleep apnoea & 2 \\
Bronchiectasis & $2(3)$ \\
Bronchiectasis & $2(3)$ \\
Heart failure &
\end{tabular}

*Includes patients previously classified by their general practitioner by the cause of nocturnal hypoventilation. were followed up in outpatient clinics where spirometry was performed in 21 patients and oximetry or measurement of arterial blood gas concentrations in 16 . Of the 11 patients in whom treatment was initiated by their general practitioner, nine were already being followed up in respiratory outpatient clinics. In five of them the mean $\mathrm{PaO}_{2}$ recorded in the notes was $9.8(2.9) \mathrm{kPa}$. Therefore only 12 out of the 41 patients with chronic obstructive lung disease who had a follow up $\mathrm{SaO}_{2}$ above $91 \%$ when breathing air met the single criterion of a $\mathrm{PaO}_{2}$ of less than $7 \cdot 3 \mathrm{kPa}$ at assessment for prescribing long term oxygen treatment.

The prescription of oxygen was appropriate in only 27 out of 58 patients who had a follow up $\mathrm{SaO}_{2}$ above $91 \%$ when breathing air (notes could not be traced for the remaining nine patients out of a total of 67). Overall, with the combination of screening oximetric results and review of $\mathrm{PaO}_{2}$ at the time of the initial prescription of oxygen in those patients who did not meet the screening requirements, 133 out of a total of 176 patients met simplified criteria for prescription of long term oxygen treatment.

\section{Discussion}

The rate of prescription of 16 per 100000 population in our study of patients prescribed long term oxygen in East London is consistent with the national rate in the United Kingdom of 8000 concentrators per 50 million population. ${ }^{9}$ In our study the initial prescription of oxygen was by a respiratory physician in over $80 \%$ of patients, most of whom had chronic obstructive lung disease. In $75 \%$ of cases the general practitioner knew that a respiratory assessment had been performed, although the results were available to them in under a third of cases. At reassessment, $76 \%$ of patients either continued to be hypoxaemic or had fulfilled the criteria for being prescribed oxygen. The median duration of installation was 19 months and $83 \%$ of patients were using oxygen for 15 hours or more. A fifth of patients admitted to still smoking. There were no significant differences in either prescription or use of oxygen between the three family health service authorities, and the larger number of concentrators prescribed in City and East London probably reflects local referral patterns.

The proportion of patients in whom formal respiratory assessments were performed compares favourably with previous surveys. In earlier studies fewer than $60 \%$ of prescriptions for long term oxygen were on the recommendation of a respiratory physician and only $43 \%$ to $63 \%$ of patients fulfilled the Department of Health criteria. ${ }^{46}$ This improvement probably reflects both local initiatives and increasingly widespread awareness of the guidelines since their introduction in 1985. Our results from East London compare favourably with results from Sweden, where $88 \%$ of patients were seen by a respiratory physician and $86 \%$ had arterial blood 
gases measured before oxygen was prescribed. ${ }^{10}$ Although general practitioners are responsible for the prescription of long term oxygen in England and Wales, our results show that they are often not informed of the results of respiratory assessments for such treatment. This situation could be improved by the use of a standardised form as a method of communication. Continued communication between respiratory physicians and general practitioners is very important in maintaining an efficient home oxygen service.

Arterial blood gas measurements are required at the time of prescription of oxygen, both to assess hypoxaemia accurately and to assess hypercapnia, but oximetry has a valuable and increasing role in screening patients for such treatment and detecting undercorrection of oxygen saturation by the concentrator. ${ }^{811} \mathrm{We}$ also used it to identify patients who were not hypoxaemic at follow up. A fifth of the patients with an oxygen saturation above $91 \%$ while breathing air at reassessment had been prescribed oxygen for nocturnal hypoventilation, in which daytime oxygen saturation could have been normal. Long term overnight oxygen treatment in patients with normal daytime $\mathrm{PaO}_{2}$ and sleep desaturation corrects arterial oxygen saturation and reduces pulmonary artery pressure, but no evidence exists that mortality is reduced. ${ }^{12}$ However, nocturnal oxygen treatment delivered by concentrator is widely used as a treatment for nocturnal hypoventilation, ${ }^{13}$ and nocturnal hypoventilation documented in sleep studies could be considered to be a further indication for installing a concentrator in the Department of Health guidelines.

Information available on the 41 patients with chronic obstructive lung disease taking oxygen who had an oxygen saturation above $91 \%$ while breathing air at reassessment showed that the criteria for prescribing oxygen were met in only 12 cases. The effect of stopping oxygen treatment in such patients, who are hypoxaemic at the time of prescription but improve with long term treatment and are no longer hypoxaemic, is unknown. ${ }^{14}$ The remaining 29 patients with chronic obstructive lung disease who had an oxygen saturation above $91 \%$ breathing air at follow up either had not been formally assessed for oxygen treatment or had a normal $\mathrm{PaO}_{2}$ at the time of prescription. Adequate assessment before prescription continues to be the most important way of ensuring appropriate use of long term oxygen.

Most patients met the criteria for the prescription of long term oxygen treatment. Only $4 \%$ of patients died within one to four months of concentrator installation compared with $13 \%$ of patients who died within the one month of concentrator prescription in a previous study. ${ }^{4}$ Patients are appropriately instructed on the use of long term oxygen and the initial problems of underuse due to poor communication with patients ${ }^{6}$ seem to have been largely overcome. Patients were able to give a reasonable account of the number of hours of oxygen they used daily, and in this study compliance and acceptability of the treatment were both excellent. Studies on whether long term oxygen treatment improves quality of life are currently in progress. Elderly patients, though less compliant with respect to hours of usage compared with younger patients, still used the concentrator for a median period of 14 hours a day and were no more likely to have problems with the treatment. Hence advanced age should not be used as a contraindication to the prescription of long term oxygen.

Most patients continue to attend hospital outpatient clinics but, despite this, adequate reassessments are still not being performed, as shown by the undercorrection $\left(\mathrm{SaO}_{2}\right.$ below $92 \%$ while using the concentrator) in $17 \%$ of patients. These patients may not be gaining maximum benefit from treatment and may require only an increased flow rate of oxygen from the concentrator (provided that they are not hypercapnic while using oxygen). Optimum follow up needs to be defined-for example, patients might benefit from screening at home with oximetry shortly after installation of a concentrator and being seen at least once in an outpatient clinic for reassessment.

The unit costs of oxygen delivered by a concentrator have fallen relative to the use of cylinders, and long term oxygen treatment for 16 hours a day currently costs $£ 500$ annually compared with $f 6000$ when oxygen is delivered by cylinders. ${ }^{15}$ In the light of changes in both costs and clinical practice of prescribing long term oxygen treatment over seven years the guidelines may need to be reviewed. There remains far more underprescription of concentrators than inappropriate prescription. ${ }^{16}$ This has implications for hypoxaemic patients with chronic obstructive lung disease who may be denied the possibility of benefit from long term oxygen and also has financial implications for the National Health Service. Nearly one million oxygen cylinders were supplied in England and Wales in $1989,{ }^{17}$ and many patients are probably still using oxygen cylinders. As oxygen taken for more than eight hours a day is more cheaply provided by oxygen concentrators, some of the costs of making long term oxygen treatment more widely available could be absorbed by rationalising the prescription of oxygen cylinders.

In conclusion, a high proportion of prescriptions conformed with the guidelines for prescription of long term oxygen. Most patients complied with treatment. However, increased communication is required between hospital doctors and general practitioners. Patients need regular reassessment to ensure that hypoxaemia is optimally corrected and that oxygen has been appropriately prescribed. Reassessment should be a condition for the continuation of treatment to maximise benefit and minimise problems. This could initially be achieved by domiciliary screening by oximetry to select the patients who would require arterial blood gas assessment and outpatient review. A formal organised home respiratory care programme is required to ensure that patients are treated optimally. 
We would like to thank the general practitioners and family health service authorities of City and East London, Waltham Forest and Redbridge, and Barking and Havering, and the Forest and Redbridge, and Barking and Havering, and the
staff of DeVilbiss Health Care for their help and cooperation with this study.

\section{Appendix}

The absolute indications for prescription, based on the entry criteria of the Medical Research Council study and the Nocturnal Oxygen Therapy Trial, ${ }^{12}$ include hyoxaemia with a $\mathrm{PaO}_{2}$ less than $7.3 \mathrm{kPa}$, hypercapnia with a $\mathrm{PaCO}_{2}$ greater than $6.0 \mathrm{kPa}, \mathrm{FEV}$ less than 1.5 litres, FVC less than 2 litres, and peripheral oedema. Relative indications include patients below the same level of hypoxaemia and spirometric characteristics but without hypercapnia and oedema. There are also categories for palliative use in the terminal phases of hypoxic lung disease and a category of other conditions causing chronic respiratory failure, such as severe kyphoscoliosis and respiratory muscle weakness.

1 Medical Research Council Working Party. Long term domiciliary oxygen therapy in chronic hypoxic cor pulmonale complicating chronic bronchitis and emphysema. Lancet 1981;i:681-6.

2 Nocturnal Oxygen Therapy Trial Group. Continuous or nocturnal oxygen therapy in hypoxemic chronic obstructive lung disease. Ann Intern Med 1980;93: 391-8.

3 Domiciliary oxygen therapy service. Drug Tariff 1990 Nov:part $\mathrm{X}$.

4 Dilworth JP, Higgs CMB, Jones PA, White RJ. Prescription of oxygen concentrators: adherence to published guidelines. Thorax 1989;44:576-8.

5 Baudouin SV, Waterhouse JC, Tahtamouni T, Smith JA,
Baxter J, Howard P. Long term domiciliary oxygen treatment for chronic respiratory failure reviewed. Thorax 1990;45:195-8.

6 Walshaw MJ, Lim R, Evans CC, Hind CRK. Prescription of oxygen concentrators for long term oxygen treatment: reassessment in one district. BMF 1988;297:1030-2.

7 Vergeret J, Tunon de Lara $M$, Douvier J, Freour $P$, Cardinaud JP, Courty G, et al. Compliance of COPD patients with long term oxygen therapy. Eur $\mathcal{F}$ Respir Dis 1986;69(suppl 146):421-5.

8 Roberts CM, Bugler JR, Melchor R, Hetzel MR, Spiro SG. Value of pulse oximetry in screening for long term oxygen therapy requirement. Eur Respir f 1993;6: oxygen

9 Howard P. Home respiratory care. Eur Respir Rev 1991;1: 563-8.

10 Strom K, Boe J. A national register for long-term oxygen therapy in chronic hypoxia: preliminary results. Eur Respir 7 1988;1:952-8.

11 Pierson DJ. Pulse oximetry versus arterial blood gas specimens in long-term oxygen therapy. Lung 1990;(suppl): 782-8.

12 Fletcher EC, Luckett RA, Goodnight-White S, Mille CC, Quian W, Costarangos-Galarza C. A double-blind trial of noctural supplemental oxygen for sleep desaturation in patients with chronic obstructive pulmonary disease and a daytime $\mathrm{PaO}_{2}$ above $60 \mathrm{~mm} \mathrm{Hg}$. Am Rev Respir Dis 1992;145:1070-6.

13 Douglas HJ, Flenley DC. Breathing during sleep in patients with obstructive lung disease. Am Rev Respir Dis 1990;141:1055-70.

14 Strom K, Boe J, The Swedish Society of Chest Medicine. Quality assessment and predictors of survival in long term domiciliary oxygen therapy. Eur Respir $f$ 1991;4: 50-8.

15 Anonymous. Home oxygen-still room for improvement. Drug Ther Bull 1990;28:99-100.

16 Williams BT, Nicholl JP. Prevalence of hypoxaemic chronic obstructive lung disease with reference to longterm oxygen therapy. Lancet 1985 ;ii:369-72.

17 Williams B, Nicholl J. Recent trends in the use of domicitiary oxygen in England and Wales. Health Trends 1991 23(4):166-7. 